\title{
A System for the Determination of Planar Lipid Bilayer Breakdown Voltage and Its Applications
}

\author{
Peter Kramar, Damijan Miklavčič, and Alenka Maček Lebar*
}

\begin{abstract}
In this paper, we focus on measurement principles used in electroporation studies on planar lipid bilayers. In particular, we point out the voltage-clamp measurement principle that has great importance when the breakdown voltage of a planar lipid bilayer is under consideration; however, it is also appropriate for the determination of other planar lipid bilayer electrical properties such as resistance and capacitance. A new experimental system that is based on the voltage-clamp measurement principle is described. With the use of a generator that can generate arbitrary-type signals, many specific shapes of a voltage signal could be generated, and therefore, the experimental system is appropriate for a broad spectrum of measurements.
\end{abstract}

Index Terms-Biomechanics, capacitance measurement, dielectric breakdown, electric field effects, isoelectric phenomena.

\section{INTRODUCTION}

$\mathbf{E}$ LECTROPORATION is characterized by the formation of structural changes within the cell plasma membrane, which are caused by the presence of an electric field. These changes, most often described as "pores," are estimated to be in the order of $1-10 \mathrm{~nm}$, increase the plasma membrane permeability, and enable transport of ions and molecules across the plasma membrane [1]. Electroporation is either reversible or irreversible. Reversible electroporation is used to introduce various substances into the cell and has practical applications in medicine, biology, and biotechnology, such as gene therapy, transdermal drug delivery, and electrochemotherapy [2]-[5]. If the cell membrane does not reseal due to a very high electric field, the electroporation is irreversible. Irreversible electroporation was suggested for liquid food and water conservation [6], [7], and tissue ablation [8], [9]. The applicability of electroporation is broad: from biotechnology and biology to medicine. The exact molecular mechanisms of the phenomenon are still not fully elucidated; however, it is believed that "pores" in the cell membrane are mostly formed by the rearrangement of lipid molecules. Therefore, artificial lipid bilayer structures in the form of planar lipid bilayers or lipid bilayer vesicles can be used as a model for experimental [10], [11] and theoretical studies of electroporation [12].

Lipid bilayer structures can be made of one type of lipid molecules or their mixtures. In more complex forms, they can

Manuscript received March 14, 2008. Current version published July 31, 2009. This work was supported by the Slovenian Research Agency under Grant P2-0249. Asterisk indicates corresponding author.

P. Kramar and D. Miklavčič are with the Faculty of Electrical Engineering, University of Ljubljana, Ljubljana SI-1000, Slovenia (e-mail: peter.kramar@fe.uni-lj.si; damijan.miklavcic@fe.uni-lj.si).

${ }^{*}$ A. M. Lebar is with the Faculty of Electrical Engineering, University of Ljubljana, Ljubljana SI-1000, Slovenia (e-mail: alenka.maceklebar@fe.unilj.si).

Digital Object Identifier 10.1109/TNB.2009.2022834

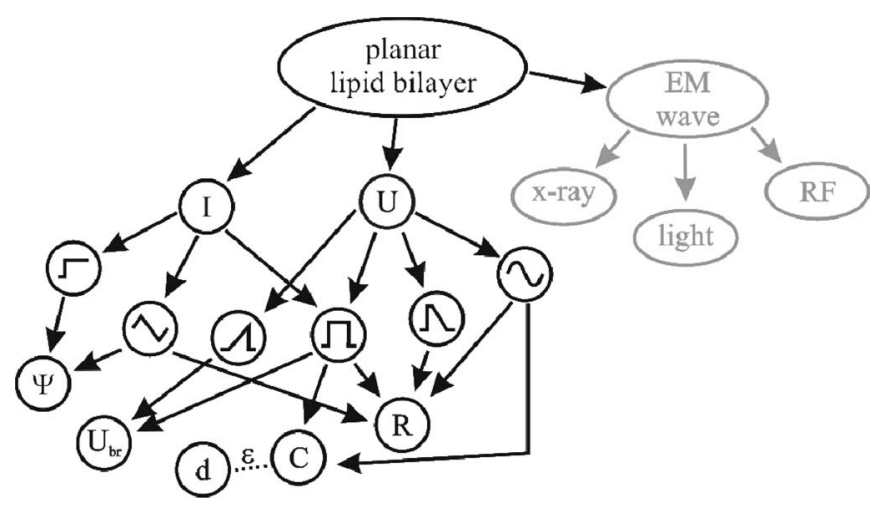

Fig. 1. Electrical properties of a planar lipid bilayer: resistance $(R)$, capacitance $(C)$, thickness $(d)$, voltage breakdown $\left(U_{\mathrm{br}}\right)$, and mass flux $(\Psi)$; these are measured with the application of current $(I)$ signal and voltage $(U)$ signal of diverse shapes. Application of electromagnetic waves is an alternative.

be composed of different lipids and proteins [13]. Lipid bilayer composition drives events during the electroporation process and determines the electrical properties of a lipid bilayer structure. According to the broadly used electric circuit model of the lipid bilayer, which consists of resistance $(R)$ and capacitance $(C)$ in parallel, resistance and capacitance are the most often measured electrical properties of the lipid bilayer. From the electroporation point of view also, the breakdown voltage $\left(U_{\mathrm{br}}\right)$, i.e., the transmembrane voltage at which the lipid bilayer brakes, and the mass flux $(\Psi)$ through the lipid bilayer are important.

Synthetic liposomes and vesicles are natural models, which mimic the geometry and topology of cell membranes. In some recent papers, it was reported that the field induces elongation of vesicles in the direction of the applied field prior to electroporation [10], and that the average rate of radius growth of the reversible pores is about five orders of magnitude smaller than that of irreversible electropores in planar lipid membranes of similar phospholipids [11].

But if the planar lipid bilayer is considered as a small fraction of the total cell membrane, it represents the simplest model for experimental studies of electroporation; especially because it is accessible from both sides.

Two measurement principles of planar lipid bilayer's properties are mainly used: the voltage-clamp method [14]-[25] and the current-clamp method [26]-[37]. In addition, due to the steadily growing use of wireless communication systems, application of electromagnetic waves is their alternative (see Fig. 1). By using different shapes of signals and frequencies, planar lipid bilayer's properties such as resistance, capacitance, thickness, voltage breakdown, and mass flux can be measured. 
In the voltage-clamp method, the voltage signal is applied to the lipid bilayer. The simplest as well as the most often used shape of the voltage signal is a square voltage pulse [14], [18], but other shapes have also been used [15], [21], [38], [39]. Some experiments were done by using two sinus-shaped voltage signals: the first with a low frequency and large amplitude, and the second with a high frequency and low amplitude that was added to the first one. In this way, lipid bilayer conductance and capacitance were determined at the same time [37].

In the current-clamp method, the current is applied to the lipid bilayer. Usually, this method is appropriate for measuring the resistance of lipid bilayer and the mass flow. It is believed that during these kinds of measurements, the lipid bilayer is more stable due to less voltage stress [27], [30]; therefore, small changes in the membrane structure can also be observed. Scalas et al. [31], for example, reported on the time course of voltage fluctuations that followed an increase of the membrane conductance due to the opening and closing of hydrophilic pores [28], [31], [40].

Under current-clamp conditions, the transmembrane voltage increases slowly; therefore, stable pores could be formed [27], [29], [32], [41]. The creation of the first pore in the planar lipid bilayer causes a fast reduction in transmembrane voltage, which decreases the probability of next pore creation [27]. Because the pore size is voltage dependent, the pore size decreases. As a consequence, the transmembrane voltage and the pore size increase again. These fluctuations of transmembrane voltage and pore size allow for maintenance of the electroporated planar lipid bilayer. Therefore, such experimental conditions are extremely useful for investigations of membrane organization at the molecular level.

Combination of electrical recording techniques with different kinds of high-frequency electromagnetic fields offers additional insights. For structure elucidation of lipid-water mesophases, small- and wide-angle X-ray scattering methods were used [42]. Hanyu et al. [43] presented an experimental system that allows measurement of current (function) and fluorescence emission (structural change) of an ion channel in the planar lipid bilayer while the membrane potential is controlled like functional relationships between the planar lipid bilayer and membrane interacting peptides. Rapid and reversible changes in photoresponsive planar lipid bilayer's electrical properties when irradiated with light were studied by Yamaguchi and Nakanishi [44]. Planar lipid bilayers have also been exposed to an RF field of about $900 \mathrm{MHz}$ according to GSM standards, and the authors reported that temperature oscillations due to the pulsed RF fields are too small to influence planar lipid bilayer's low-frequency behavior [45].

In this paper, we focus on the voltage-clamp measurement principle. We explain advantages and disadvantages of this method and describe its measuring system requirements. We present a new measuring system that has broad applicability; the new system allows generation of arbitrary waveforms, and therefore, many existing experimental procedures can be repeated and completely new measurements can be planned.

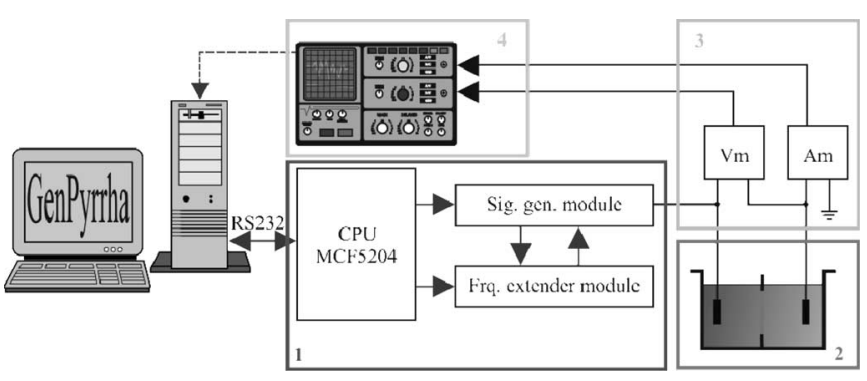

Fig. 2. Experimental system: 1) microprocessor board with MCF5024 processor and two modules. The signal generator module generates arbitrary signals. The frequency extender module is realized in the programmable integrated circuit (FPGA) and is used for frequency extension; 2) chamber for forming planar lipid bilayer and two $\mathrm{Ag}-\mathrm{AgCl}$ electrodes; 3 ) modules for current and voltage amplification; 4) oscilloscope for data collection and storage.

\section{EXPERIMENTAL SYSTEM}

The system consists of a signal generator, a Teflon chamber where the planar lipid bilayer is prepared, a device for voltage and current measurement and storage. Fig. 2 presents a typical structure of the experimental system: a signal generator (see Fig. 2-1), a chamber for forming planar lipid bilayers and electrodes (see Fig. 2-2), voltage and current amplifiers (see Fig. 2-3), and an oscilloscope for data storage (see Fig. 2-4).

\section{A. Signal Generator}

Signal generator is a generator of an arbitrary type. It provides voltage amplitudes from -5 to $+5 \mathrm{~V}$. It is controlled by customdesigned software, specially designed for drawing the arbitrary voltage signals that are used for membrane electroporation. In the program, the gain of the output amplifier and the way of triggering can be selected as single, continuous, or burst type. The signal can be either monopolar or bipolar, and a different number of pulses can be generated. The last but not least part of the signal generator is an analog switch. The switch disconnects the output of the signal generator and switches one electrode to the 1-M $\Omega$ resistor. The switch is fast: it turns off the signal generator in $2 \mathrm{~ns}$. In this way, the system discharge voltage, as well as the capacitance of the lipid bilayer, is measured. The signal time resolution is $860 \mathrm{~ns}$ or $1.17 \mathrm{MHz}$; however, lower and higher frequencies can also be obtained.

For higher frequencies applications, a special circuit for frequency extensions that consists of a programmable integrated circuit [field-programmable gate array (FPGA)] is added. The highest sampling frequency is $33 \mathrm{MHz}$. Lower frequencies can be selected by inner decades that are programmed in the programmable integrated circuit and separated by a 7-bit binary counter.

\section{B. Voltage and Current Amplification, and Storage}

Two $\mathrm{Ag}-\mathrm{AgCl}$ electrodes (IVM, USA), one on each side of the planar lipid bilayer, are immersed into a salt solution. The transmembrane voltage is measured by LeCroy differential amplifier 1822. The same electrodes are also used to measure the transmembrane current. An electronic circuit transforms the 

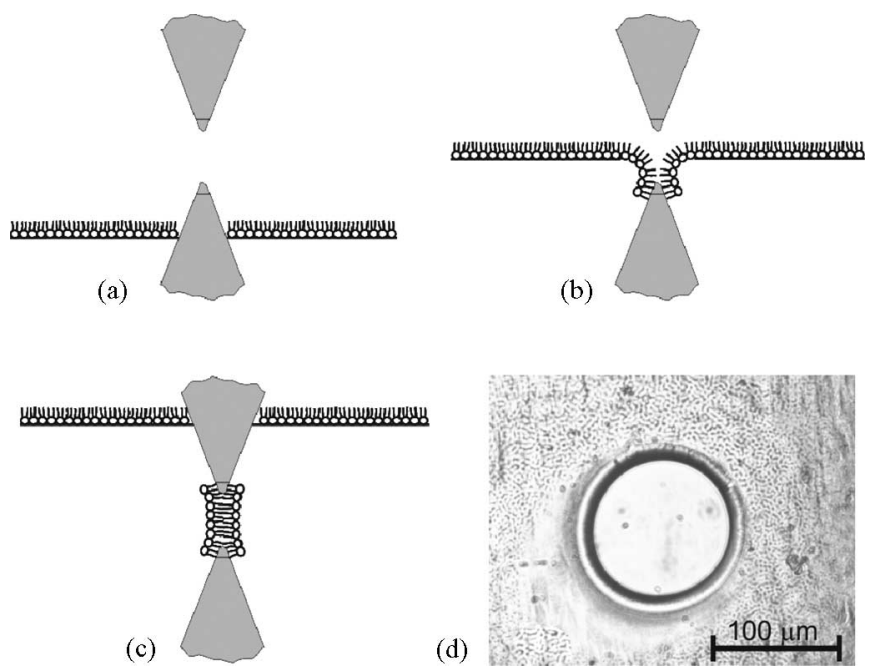

(d)

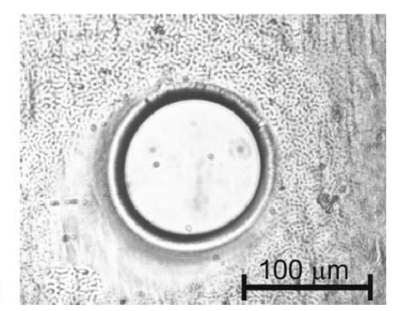

Fig. 3. Montal-Müeller method for planar lipid bilayer formation [46]. (a) Layer of lipid molecules on the salt solution. (b) Level of the salt solution is slowly raised above the hole. (c) Planar lipid bilayer is made on the hole. (d) Round hole in a Teflon sheet made by electrical discharge. The image of the hole was captured by Olympus DP 10 Camera and Olympus DP soft programme through Olympus CK 40 microscope at the $20 \times$ magnification. The diameter of the hole $(105 \mu \mathrm{m})$ was determined by Olympus DP-soft programme measuring tools.

transmembrane current into voltage, so that $1 \mathrm{~V}$ corresponds to $1 \mathrm{~mA}$ of a transmembrane current.

Both signals are stored by the oscilloscope LeCroy Waverunner-2 354M in the MATLAB format. All sampled signals are analyzed in MATLAB software after experiments.

\section{Chamber}

The chamber is made of Teflon. Teflon is highly resistant to chemicals, and therefore, it may be cleaned with aggressive chemicals such as sulfuric acid $\left(\mathrm{H}_{2} \mathrm{SO}_{4}\right)$. Between experiments the chamber is soaked in $\mathrm{H}_{2} \mathrm{SO}_{4}$. The chamber consists of two parts; each part is a cubed reservoir with a volume of $5.3 \mathrm{~cm}^{3}$. Between the reservoirs a 25.40- $\mu \mathrm{m}$-thin Teflon sheet is inserted. A round hole [see Fig. 3(d)] was made in the Teflon sheet by electrical discharge. The diameter of the hole was measured (105 $\mu \mathrm{m})$ and the area of the hole was calculated $\left(8.66 \mathrm{~nm}^{2}\right)$. The lipid bilayer is formed on the hole by a folding method given by Montal and Müeller [see Fig. 3(a)-(c)] [46]. This method was selected due to its fast rebuilding ability of a planar lipid bilayer after each breakdown. The membrane was assumed to span $90 \%$ of the area of the hole, while the remaining $10 \%$ is occupied by the torus of solvent that supports the membrane.

The salt solution is poured in both reservoirs through special channels. The channels have a diameter of $0.8 \mathrm{~mm}$ and lies below $50^{\circ}$ with respect to the bottom of the reservoirs. Through each channel, a pipeline is inserted into each reservoir. The level of salt solution in the reservoir is regulated by using a syringe filled with salt solution, which is attached to the outer side of the pipeline.
TABLE I

SPECIFIC MEMBRANE CAPACITANCES

\begin{tabular}{lll}
\hline \hline \multicolumn{1}{c}{ Lipid mixtures } & \multicolumn{1}{c}{$\mathrm{C}\left(\mu \mathrm{F} / \mathrm{CM}^{2}\right)$} & $\mathrm{N}$ \\
\hline POPC & $0.5 \pm 0.1$ & 33 \\
POPC $+1 \mu \mathrm{M} \mathrm{C}_{12} \mathrm{E}_{8}$ & $0.21 \pm 0.02$ & 37 \\
POPS:POPC (3:7) & $0.29 \pm 0.07$ & 42 \\
\hline \hline
\end{tabular}

Specific membrane capacitances $(C)$ that have been measured for three lipid mixtures. Values given are mean \pm standard deviation. The number of measurements $(N)$ in each experimental group is given in the third column.

\section{Chemicals}

The results presented in this paper were obtained by chemicals described in this section. Our experiments were done using the salt solution prepared of 0.1 molar $\mathrm{KCl}$ (MERCK, Germany) and 0.01 molar 4-(2-hydroxyethyl)-1-piperazineethanesulfonic acid (Hepes) (MERCK, Germany) in the same proportion. Some droplets of 1 molar $\mathrm{NaOH}$ (MERCK, Germany) were added to obtain a $\mathrm{pH}$ value of 7.4.

Synthetic lipids 1-palmitoyl-2-oleoyl-sn-glycero-3phosphocholine (POPC) and 1-palmitoyl-2-oleoyl-sn-glycero3-[phospho-L-serine] (POPS) were used in our experiments. Both are common parts of experimental structures (lipid bilayers and vesicles) and mimic at least one important constituent of the cell membrane-glycerophospholipids. They were obtained in powder form (Avanti Polar Lipids, Inc., ZDA), and melted in the solution of hexane and ethanol (Riedel-de Haën, Germany) in a ratio of 9:1. Octaethyleneglycol mono- $n$-dodecyl ether $\left(\mathrm{C}_{12} \mathrm{E}_{8}\right)$ was obtained in crystal form (Fluka, Switzerland). The mixture of hexadecane and pentane (Merck, Germany) in a ratio of 3:7 was used for torus forming.

\section{Measurement Principles and Properties of Planar LIPID BILAYERS}

The system described has been tested with different experimental protocols. Some of them were already proposed by other authors. We have determined the capacitance of lipid bilayers [18] and the breakdown voltage of planar lipid bilayers using rectangular pulses [14], [21], [24] as well as linear rising signals [38].

\section{A. Capacitance of Lipid Bilayer}

The capacitance $(C)$ is a parameter that is considered to be the best tool for probing the stability and formal goodness of planar lipid bilayers. Using the system described, $C$ is determined by the discharge method [18]. The data measured for each planar lipid bilayer mixture are presented in Table I.

\section{B. Conductance During Electroporation}

Resistance $(R)$ or conductance $(G)$ as an electrical property of planar lipid bilayers can be measured only during the application of voltage or current signals. Measurement of the current requires high sensitivity in the range of nanoamperes.

When a rectangular voltage pulse of a high amplitude is applied on the planar lipid bilayer, electroporation occurs and the conductance of the lipid bilayer increases considerably (see 


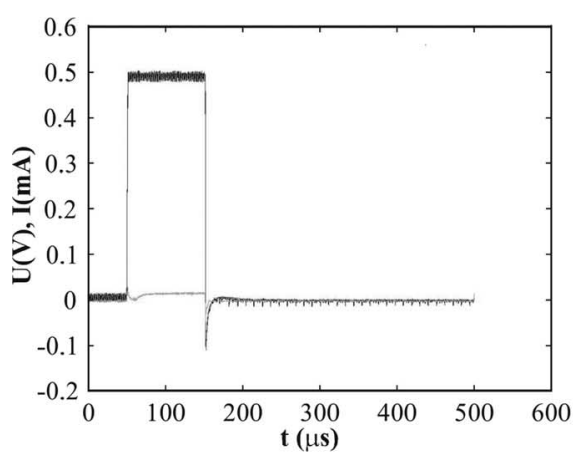

(a)

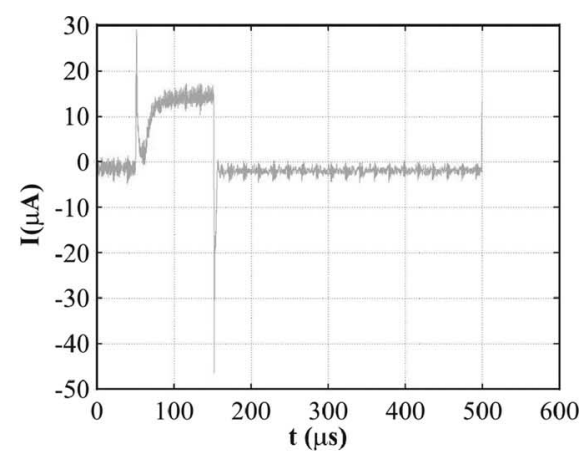

(b)

Fig. 4. Electroporation of the planar lipid bilayer. (a) Applied square signal and measured transmembrane current. (b) Enlargement of the transmembrane current during the voltage pulse.

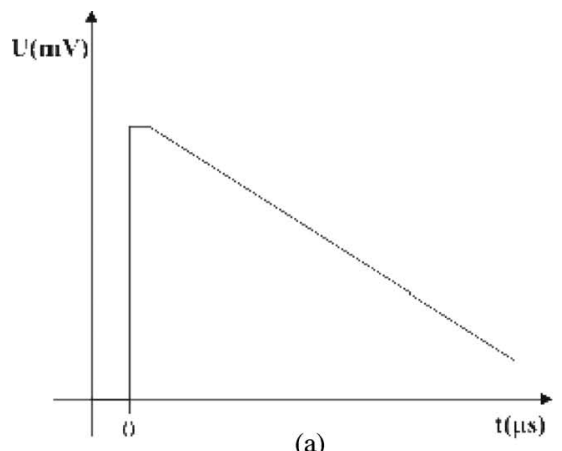

(a)

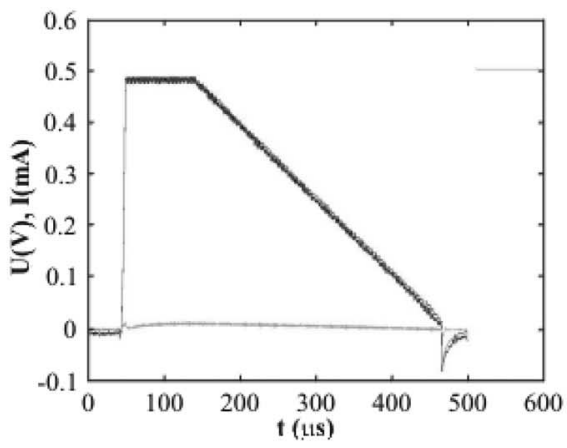

(c)

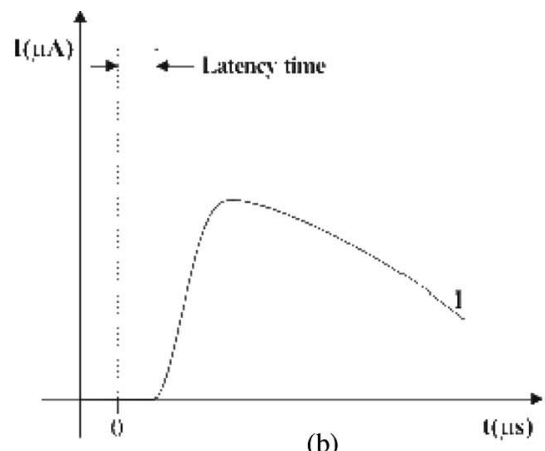

(b)

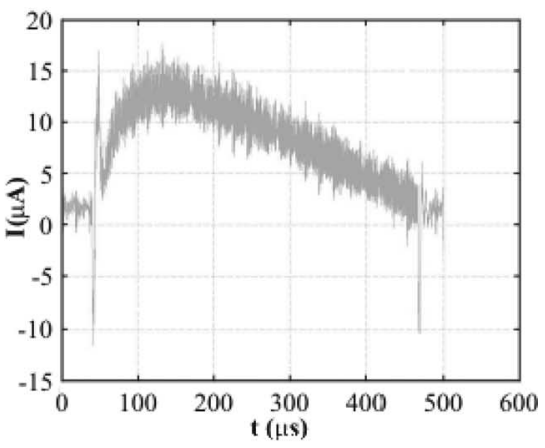

(d)

Fig. 5. (a) Voltage signal composed of an initial rectangular pulse and a negative slope ramp [15]. (b) Transmembrane current. $I$ is a typical trace of the transmembrane current during the electroporation. (c) and (d) Corresponding results of our experiments.

Fig. 4). Consequently, current through the planar lipid bilayer also increases by several orders of magnitude, as shown in Fig. 4. Due to the constant voltage amplitude during the pulse, the dynamic conductance $[G(t)]$ is determined by

$$
G(t)=\frac{i(t)}{u(t)}
$$

where $i(t)$ is the current measured through the planar lipid bilayer and $u(t)$ is the applied voltage.

Sharma et al. have used a special waveform for measuring the dynamic conductance of the lipid bilayer during the electroporation [15]. The applied signal is composed of an initial rectangular pulse and a negative-sloped ramp. Such a pulse protocol allows longer measurement of membrane conductance. Due to the fact that with the system described in this paper an arbitrary shape of the voltage signal can be provided to the electrodes, our system is applicable for such measurements also (see Fig. 5).

\section{Breakdown Voltage of the Planar Lipid Bilayer}

Breakdown voltage $\left(U_{\mathrm{br}}\right)$ is one of the most important properties of a lipid bilayer when biomedical and biotechnological applications of electroporation are under consideration.

Mostly, the breakdown voltage of the planar lipid bilayer is determined by applying a rectangular voltage pulse (10 $\mu \mathrm{s}-10$ s). The amplitude of the voltage pulse is incremented in small steps until the breakdown of the bilayer is reached [14]. First, the voltage pulse charges up the planar lipid bilayer. Above the critical voltage $\left(U_{\mathrm{br}}\right)$, defects are caused in the planar lipid bilayer 


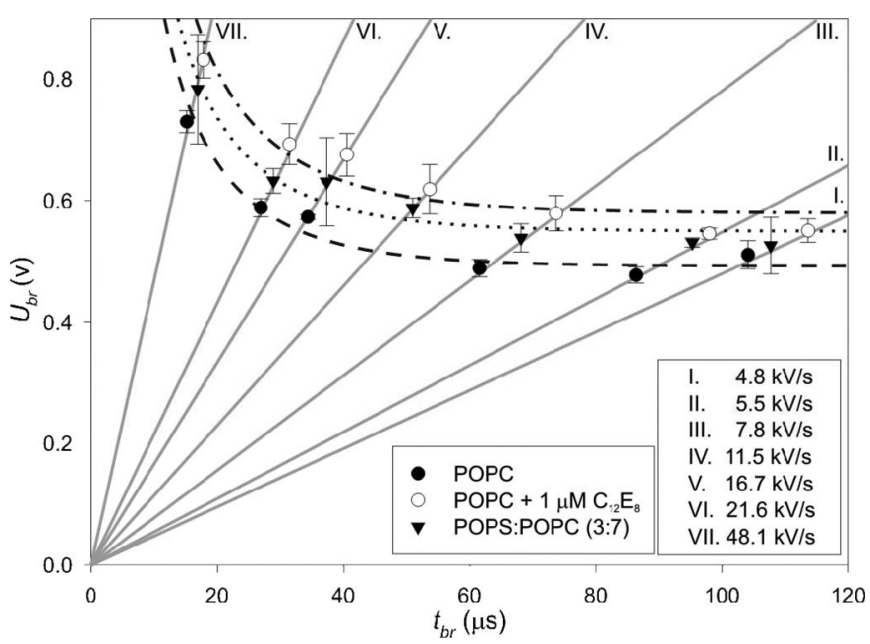

Fig. 6. Breakdown voltage $\left(U_{\mathrm{br}}\right)$ (dots) of planar lipid bilayers with different chemical composition as a function of lifetime $t_{\mathrm{br}}$. The gray lines show seven different slopes of the applied linear rising voltage signal. Dash, dotted, and dash-dotted curves represent a two-parameter curve fitted to data (2).

allowing an increase of the transmembrane current. Usually, the membrane collapses thereafter.

Using the rectangular voltage pulse measuring protocol, the number of applied voltage pulses is not known in advance and each planar lipid bilayer is exposed to a voltage stress many times. Such a pretreatment of the planar lipid bilayer affects its stability and consequently the determined breakdown voltage of the planar lipid bilayer [24]. Another approach for the breakdown voltage determination was suggested by our group [38]. Using a linear rising signal, the breakdown voltage of each planar lipid bilayer is determined by a single voltage exposure.

The slope of the linear rising signal and the peak voltage of the signal have to be selected in advance. Breakdown voltage is defined as the voltage at the moment $t_{\mathrm{br}}$ when a sudden increase of the transmembrane current is observed. Time $t_{\mathrm{br}}$ was defined as the lifetime of the planar lipid bilayer at a chosen slope of the linear rising signal (see Fig. 6). Due to the already known experimental evidence that the planar lipid bilayer lifetime depends on the applied voltage [14], [47] and the planar lipid bilayer breakdown voltage depends on the planar lipid bilayer pretreatment [24], $U_{\mathrm{br}}$ and $t_{\mathrm{br}}$ were measured at six or seven different slopes. Indeed, the lifetime of the planar lipid bilayer depends on the slope of the linear rising voltage signal, and also the breakdown voltage is a function of the slope of the linear rising voltage signal; it increases with the increasing slope. Therefore, using nonlinear regression, a two-parameter curve was fitted to the data,

$$
U=\frac{a}{1-e^{-t / b}}
$$

where $U$ is the $U_{\mathrm{br}}$ measured at different slopes, $t$ is the corresponding $t_{\mathrm{br}}$, and $a$ and $b$ are the parameters. Parameter $a$ is an asymptote of the curve that corresponds to minimal breakdown voltage $U_{\mathrm{brMIN}}$ for a specific planar lipid bilayer chemical composition. Parameter $b$ governs the inclination of the curve.

The results obtained with the described measuring protocol are presented in Fig. 6. $U_{\mathrm{br}}$ and $t_{\mathrm{br}}$ of POPC, POPC $+1 \mu \mathrm{M}$
$\mathrm{C}_{12} \mathrm{E}_{8}$, and POPS:POPC (3:7) planar lipid bilayers have been measured, and $U_{\mathrm{brMIN}}$ for each planar lipid bilayer chemical composition has been calculated: $U_{\mathrm{brMIN}}$ is $0.49,0.58$, and $0.55 \mathrm{~V}$, respectively.

\section{DISCUSSION}

In this paper, we focused on measurement principles used in electroporation studies on planar lipid bilayers. We pointed out the voltage-clamp measurement principle that has great importance when the breakdown voltage of planar lipid bilayers is under consideration; however, it is also appropriate for the determination of other planar lipid bilayer electrical properties such as resistance and capacitance. A new experimental system that is based on the voltage-clamp measurement principle was described. Due to the signal generator of an arbitrary pulse shape, many special shapes of the voltage signal could be generated, and therefore, the experimental system is appropriate for a broad spectrum of measurements. Values of planar lipid bilayer capacitance, dynamic conductance during irreversible breakdown of the planar lipid bilayer, and lipid bilayer breakdown voltage are given.

The most common and simple method for measuring planar lipid bilayer capacitance is the discharge pulse method, which was described in detail by Sharma et al. [15], and Benz and Janko [18]. The method is easily applied using the described system. Due to the fact that the capacitance of the planar lipid bilayer depends on the bilayer composition as well as on the concentration of the salt solution [39], many different values can be found in scientific papers [48]; the results given in Table I are in line with previous measurements of planar lipid bilayer capacitance. The voltage-clamp method has also been used by Gallucci et al., who have described a measurement system where two sinus signals with frequencies $1 \mathrm{~Hz}$ and $1 \mathrm{kHz}$ are mixed and applied to the planar lipid bilayer. The amplitude and phase of both signals govern the planar lipid bilayer capacitance and resistance simultaneously [39]. Such continuous monitoring of $C$ may prove useful in tracking planar lipid bilayer electrical properties that depend on the lipid composition and incorporated nonphospholipids substances. This method allows measuring of electrical properties of nonpermeabilized planar lipid bilayers and during the process of defect formation-electroporation. Capacitance of a planar lipid bilayer can also be determined by a triangular voltage signal [26] that is controlled by the regulation system that includes a charged planar lipid bilayer as a part. In this way, the period of the triangular voltage signal is related to the capacitance of the planar lipid bilayer.

Conductance measurements were presented by tracking dynamic conductance during the irreversible breakdown of the planar lipid bilayer (see Fig. 4). Measurement of fluctuation and transient pores in the planar lipid bilayer requires highsensitivity current measurements in the range of nanoamperes. Melikov et al. [22] monitored fluctuations in the planar lipid bilayer conductance induced by applying a voltage step of sufficiently high amplitude. They demonstrated that the amplitude of fluctuations varied in a rather broad interval (form 150 to 
$1500 \mathrm{pS}$ ), and they related it to the formation of local conductive defects-hydrophilic pores.

Breakdown voltage $\left(U_{\mathrm{br}}\right)$ is one of the most important properties of a lipid bilayer when biomedical and biotechnological applications of electroporation are under consideration. $U_{\mathrm{br}}$ depends on the composition of the planar lipid bilayer, i.e., the type of lipid hydrophilic chain and nonphospholipid substances. By a rectangular voltage pulse, $U_{\mathrm{br}}$ of planar lipid bilayers made of many different compositions was determined [48]. Meier et al. have reported that palmitoyl-oleoyl (PO) membranes require $\sim 100 \mathrm{mV}$ smaller breakdown voltages compared to diphytanoyl (DPh) membranes [16]. Incorporation of nonphospholipid substances into planar lipid bilayers changes $U_{\mathrm{br}}$ as well. The effect is a consequence of the surfactant molecular shape acting to change the spontaneous curvature of the membrane, which is especially important during the defects formation process [12], [49], [50].

$U_{\mathrm{br}}$ has been measured either by the voltage-clamp or the current-clamp method using a rectangular, triangular, or step shape of the signal (see Fig. 1). However, voltage-clamp methods provide great stress to the planar lipid bilayer and hence, are not appropriate for observation of small defects-pores in the membrane structure. Namely, the strong electric field caused by the applied voltage usually generates a diverse population of pores in the planar lipid bilayer [22] and eventually planar lipid bilayer destruction. By means of a linear rising signal, $U_{\mathrm{br}}$ is determined by single voltage exposure that applies lower voltage stress on the planar lipid bilayer than during the pulse measuring protocol.

Therefore, the new experimental system, which is based on the voltage-clamp measurement principle and allows generation of arbitrary waveforms, can be a great contribution to the planning of new experimental procedures and makes it easier to perform the existing procedures.

\section{REFERENCES}

[1] E. Neumann, S. Kakorin, and K. Toensig, "Fundamentals of electroporative delivery of drugs and genes," Bioelectrochem. Bioenerg., vol. 48, pp. 3-16, 1999.

[2] M. Cemazar, D. Miklavcic, L. M. Mir, J. Belehradek Jr., M. Bonnay, D. Fourcault, and G. Sersa, "Electrochemotherapy of tumours resistant to cisplatin: A study in a murine tumour model," Eur. J. Cancer, vol. 37, pp. 1166-1172, 2001

[3] A. R. Denet and V. Preat, "Transdermal delivery of timolol by electroporation through human skin," J. Controlled Release, vol. 88, pp. 253-262, 2003.

[4] D. Ferber, "Gene therapy. Safer and virus-free?," Science, vol. 294, pp. $1638-1642,2001$

[5] L. M. Mir and S. Orlowski, "Mechanisms of electrochemotherapy," $A d v$. Drug Del. Rev., vol. 35, pp. 107-118, 1999.

[6] M. C. Vernhes, A. Benichou, P. Pernin, P. A. Cabanes, and J. Teissie, "Elimination of free-living amoebae in fresh water with pulsed electric fields," Water Res., vol. 36, pp. 3429-3438, 2002.

[7] G. W. Gould, "Biodeterioration of foods and an overview of preservation in the food and dairy industries," Int. Biodeter. Biodegrad., vol. 34, pp. 267-277, 1995.

[8] B. Rubinsky, G. Onik, and P. Mikus, "Irreversible electroporation: A new ablation modality-clinical implications," Technol. Cancer Res. Treatment, vol. 6, pp. 37-48, 2007.

[9] R. V. Davalos, L. M. Mir, and B. Rubinsky, "Tissue ablation with irreversible electroporation," Ann. Biomed. Eng., vol. 33, pp. 223-231, 2005.
[10] N. Asgharian and Z. A. Schelly, "Electric field-induced transient birefringence and light scattering of synthetic liposomes," Biochim. Biophys. Acta, vol. 1418, pp. 295-306, 1999.

[11] N. M. Correa and Z. A. Schelly, "Electroporation of unilamellar vesicles studied by using a pore-mediated electron-transfer reaction," Langmuir, vol. 14, pp. 5802-5805, 1998.

[12] M. Fošnarič, V. Kralj-Iglič, K. Bohinc, A. Iglič, and S. May, "Stabilisation of pores in lipid bilayers by anisotropic inclusions," J. Phys. Chem. B, vol. 107, pp. 12519-12526, 2003.

[13] H. T. Tien and A. L. Ottova, "The lipid bilayer concept and its experimental realisation: From soap bubbles, kitchens sink, to bilayer lipid membranes," J. Membrane Sci., vol. 189, pp. 83-117, 2001.

[14] G. C. Troiano, L. Tung, V. Sharma, and K. J. Stebe, "The reduction in electroporation voltages by the addition of surfactant to planar lipid bilayer," Biophys. J., vol. 75, pp. 880-888, 1998.

[15] V. Sharma, K. U. Maheswari, J. C. Murphy, and L. Tung, "Poloxamer 188 decreases susceptibility of artificial lipid membranes to electroporation," Biophys. J., vol. 71, pp. 3229-3241, 1996.

[16] W. Meier, A. Graff, A. Diederich, and M. Winterhalter, "Stabilization of planar lipid membranes: A stratified layer approach," Phys. Chem. Chem. Phys., vol. 2, pp. 4559-4562, 2000.

[17] A. Diederich, G. Bahr, and M. Winterhalter, "Influence of surface charges on the rapture of black lipid membranes," Phys. Rev. E, vol. 58, pp. 48834889, 1998.

[18] R. Benz and K. Janko, "Voltage-induced capacitance relaxation of lipid bilayer membranes; effects on membrane composition," Biochim Biophys. Acta, vol. 455, pp. 721-738, 1976.

[19] J. Vargas, J. M. Alarcon, and E. Rojas, "Displacement currents associated with the insertion of alzheimer disease amyloid (beta)-peptide into planar bilayer membranes," Biophys. J., vol. 79, pp. 934-944, 2000

[20] C. Wilhelm, M. Winterhalter, U. Zimmermann, and R. Benz, "Kinetics of pore size during irreversible electrical breakdown of lipid bilayer membranes," Biophys. J., vol. 64, pp. 121-128, 1993.

[21] R. Benz, F. Beckers, and U. Zimmermann, "Reversible electrical breakdown of lipid bilayer membranes: A charge-pulse relaxation study," $J$. Membrane Biol., vol. 48, pp. 181-204, 1979.

[22] K. C. Melikov, V. A. Frolov, A. Shcherbakov, A. V. Samsonov, Y. A Chizmadzhev, and L. V. Chernomordik, "Voltage-induced nonconductive pre-pores and metastable single pores in unmodified planar lipid bilayer," Biophys. J., vol. 80, pp. 1829-1836, 2001.

[23] R. W. Glaser, S. L. Leikin, L. V. Chernomordik, V. F. Pastushenko, and A. I. Sokirko, "Reversible electrical breakdown of lipid bilayers: Formation and evolution of pores," Biochim. Biophys. Acta, vol. 940, pp. 275287, 1988.

[24] I. G. Abidor, V. B. Arakelyan, L. V. Chernomordik, Y. A. Chizmadzhev, V. F. Pastushenko, and M. R. Tarasevich, "246-Electric breakdown of bilayer lipid membranes I. The main experimental facts and their qualitative discussion," Bioelectrochem. Bioenerg., vol. 6, pp. 37-52, 1979.

[25] A. N. Chanturiya, "Detection of transient capacitance increase associated with channel formation in lipid bilayers," Biochim. Biophys. Acta, vol. 1026, pp. 248-250, 1990.

[26] S. Kalinowski and Z. Figaszewski, "A new system for bilayer lipid membrane capacitance measurements: Method, apparatus and applications," Biochim. Biophys. Acta, vol. 1112, pp. 57-66, 1992.

[27] S. Koronkiewicz, S. Kalinowski, and K. Bryl, "Programmable chronopotentiometry as a tool for the study of electroporation and resealing of pores in bilayer lipid membranes," Biochim. Biophys. Acta, vol. 1561, pp. 222-229, 2002.

[28] A. Ridi, E. Scalas, M. Robello, and A. Gliozzi, "Linear response of a fluctuating lipid bilayer," Thin Solid Films, vol. 327-329, pp. 796-799, 1998.

[29] M. Robello and A. Gliozzi, "Conductance transition induced by an electric field in lipid bilayers," Biochim. Biophys. Acta, vol. 982, pp. 173-176, 1989.

[30] A. Ridi, E. Scalas, and A. Gliozzi, "Noise measurements in bilayer lipid membranes during electroporation," Eur. Phys. J. E, vol. 2, pp. 161-168, 2000.

[31] E. Scalas, A. Ridi, M. Robello, and A. Gliozzi, "Flicker noise in bilayer lipid membranes," Europhys. Lett., vol. 43, pp. 101-105, 1998.

[32] I. Genco, A. Gliozzi, A. Relini, M. Robello, and E. Scalas, "Electroporation in symmetric and asymmetric membranes," Biochim. Biophys. Acta vol. 1149, pp. 10-18, 1993.

[33] E. Pescio, A. Ridi, and A. Gliozzi, "A picoampere current generator for membrane electroporation," Rev. Sci. Instrum., vol. 71, pp. 1740-1744, 2000. 
[34] M. Robello, M. Fresia, L. Maga, A. Grasso, and S. Ciani, "Permeation of divalent cations through (alpha)-latrotoxin channels in lipid bilayers: Steady-state current-voltage relationship," J. Membrane Biol., vol. 95, pp. 55-62, 1987.

[35] S. Kalinowski and Z. Figaszewski, "A four-electrode system for measurement of bilayer lipid membrane capacitance," Meas. Sci. Technol, vol. 6, pp. 1034-1049, 1995.

[36] S. Kalinowski and Z. Figaszewski, "A four-electrode potentiostatgalvanostat for studies of bilayer lipid membranes," Meas. Sci. Technol., vol. 6, pp. 1050-1055, 1995.

[37] S. Micelli, E. Gallucci, and V. Ricciarelli, "Studies of mitochondrial porin incorporation parameters and voltage-gated mechanism with different black lipid membranes," Bioelectrochemistry, vol. 52, pp. 63-75, 2000.

[38] P. Kramar, D. Miklavčič, and A. M. Lebar, "Determination of lipid bilayer breakdown voltage by means of linear rising signal," Bioelectrochemistry, vol. 70, pp. 23-27, 2007.

[39] E. Gallucci, S. Micelli, and G. Monticelli, "Pore formation in lipid bilayer membranes made of phosphatidylinositol and oxidized cholesterol followed by means of alternating current," Biophys. J., vol. 71, pp. 824-831, 1996.

[40] S. Kalinowski, G. Ibron, K. Bryl, and Z. Figaszewski, "Chronopotentiometric studies of electroporation of bilayer lipid membranes," Biochim. Biophys. Acta, vol. 1396, pp. 204-212, 1998.

[41] S. Koronkiewicz, S. Kalinowski, and K. Bryl, "Programmable chronopotentiometry as a tool for the study of electroporation and resealing of pores in bilayer lipid membranes," Biochim. Biophys. Acta, vol. 1561, pp. 222-229, 2004.

[42] A. Blume, "Lipids," in Bioelectrochemistry of Membranes, D. Waltz, J. Teissié, and G. Milazzo, Eds. Basel, Switzerland: Birkhauser, 2004, pp. 24-61.

[43] Y. Hanyu, T. Yamada, and G. Matsumoto, "Simultaneous measurement of spectroscopic and physiological signals from a planar bilayer system: Detecting voltage-dependent movement of a membrane-incorporated peptide," Biochemistry, vol. 37, pp. 15376-15382, 1998.

[44] H. Yamaguchi and H. Nakanishi, "Characterization of the preparation process and photochemical control of electrical properties of bilayer lipid membranes containing azobenzene chromophores," Biochim. Biophys. Acta, vol. 1148, pp. 179-184, 1993.

[45] T. F. Eibert, M. Alaydrus, F. Wiczewski, and V. W. Hansen, "Electromagnetic and thermal analysis for lipid bilayer membranes exposed to RF fields," Trans. Biomed. Eng., vol. 46, pp. 1013-1021, 1999.

[46] M. Montal and P. Müeller, "Formation of bimolecular membranes from lipid monolayers and study their electrical properties," Proc. Nat. Academy Sci. USA, vol. 69, pp. 3561-3566, 1972.

[47] A. Maček-Lebar, G. C. Troiano, L. Tung, and D. Miklavčič, "Inter-pulse interval between rectangular voltage pulses affects electroporation threshold of artificial lipid bilayers," IEEE Trans. Nanobiosci., vol. 1, no. 3, pp. 116-120, Sep. 2002.

[48] M. Pavlin, T. Kotnik, D. Miklavčič, P. Kramar, and A. M. Lebar, "Electroporation of planar lipid bilayers and membranes," in Advances in Planar Lipid Bilayers and Liposomes, vol. 6, A. L. Liu, Ed. Amsterdam, The Netherlands: Elsevier, 2008, pp. 165-226.
[49] B. Babnik, D. Miklavčič, M. Kandušer, H. Hagerstrand, V. Kralj-Iglič, and A. Iglič, "Shape transformation and burst of giant POPC unilamellar liposomes modulated by non-ionic detergent C12E8," Chem. Phys. Lipids, vol. 125 , pp. 123-138, 2003.

[50] B. Mavcic, B. Babnik, A. Iglic, M. Kanduser, T. Slivnik, and V. Kralj-Iglic, "Shape transformation of giant phospholipid vesicles at high concentrations of C12E8," Bioelectrochemistry, vol. 63, pp. 183-187, 2004.

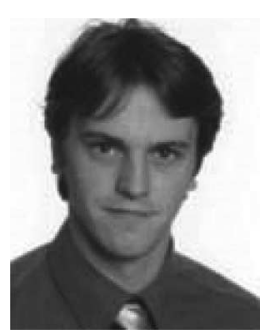

Peter Kramar was born in Kranj, Slovenia, in 1977. He received the B.Sc. and M.Sc. degrees in electrical engineering from the Faculty of Electrical Engineering, University of Ljubljana, Ljubljana, Slovenija, in 2003 and 2005, respectively.

$\mathrm{He}$ is currently a Teaching Assistant with the University of Ljubljana. His current research interests include electroporation of planar lipid bilayers.

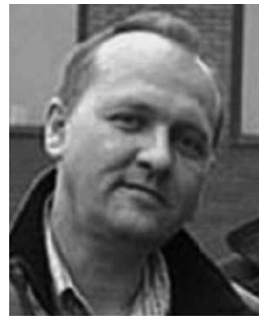

Damijan Miklavčič was born in Ljubljana, Slovenia, in 1963. He received the Ph.D. degree in electrical engineering from the University of Ljubljana, Ljubljana.

$\mathrm{He}$ is currently a Professor with the Faculty of Electrical Engineering, University of Ljubljana, where he is also the Head of the Laboratory of Biocybernetics, and is actively engaged in the field of biomedical engineering. His current research interests include electroporation-assisted drug and gene delivery, including cancer treatment by means of electrochemotherapy, tissue oxygenation, and modeling.

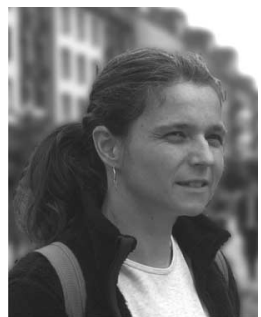

Alenka Maček Lebar was born in Ljubljana, Slovenia, in 1967. She received the M.Sc. and Ph.D degrees in electrical engineering from the University of Ljubljana, Ljubljana, in 1995 and 1999, respectively.

She is currently an Assistant Professor with the Faculty of Electrical Engineering, University of Ljubljana. Her current research interests include the biomedical engineering field, especially the field of electroporation. 